\title{
Mucoadhesive Hydrogel Gargle
}

National Cancer Institute

\section{Source}

National Cancer Institute. Mucoadhesive Hydrogel Gargle. NCI Thesaurus. Code C127117.

An oral rinse composed of a viscous, mucoadhesive hydrogel, with potential protective and antimucositis activities. Upon gargling with the oral viscous mucoadhesive hydrogel formulation in the oral cavity, the hydrogel forms a protective barrier over the oral mucosa, which prevents inflammation of the mucosal membranes and may decrease chemotherapy- and/or radiation-induced oral mucositis. 